\title{
Derivation of inner magnetospheric electric field (UNH-IMEF) model using Cluster data set
}

\author{
H. Matsui ${ }^{1}$, P. A. Puhl-Quinn ${ }^{1}$, V. K. Jordanova ${ }^{2}$, Y. Khotyaintsev ${ }^{3}$, P.-A. Lindqvist ${ }^{4}$, and R. B. Torbert ${ }^{1}$ \\ ${ }^{1}$ Space Science Center, University of New Hampshire, Durham, NH 03824, USA \\ ${ }^{2}$ Los Alamos National Laboratory, Los Alamos, NM 87545, USA \\ ${ }^{3}$ Swedish Institute of Space Physics, 751-21 Uppsala, Sweden \\ ${ }^{4}$ Royal Institute of Technology, 100-44 Stockholm, Sweden
}

Received: 2 April 2008 - Revised: 15 July 2008 - Accepted: 15 August 2008 - Published: 23 September 2008

\begin{abstract}
We derive an inner magnetospheric electric field (UNH-IMEF) model at $L=2-10$ using primarily Cluster electric field data for more than 5 years between February 2001 and October 2006. This electric field data set is divided into several ranges of the interplanetary electric field (IEF) values measured by ACE. As ring current simulations which require electric field as an input parameter are often performed at $L=2-6.6$, we have included statistical results from ground radars and low altitude satellites inside the perigee of Cluster in our data set $(L \sim 4)$. Electric potential patterns are derived from the average electric fields by solving an inverse problem. The electric potential pattern for small IEF values is probably affected by the ionospheric dynamo. The magnitudes of the electric field increase around the evening local time as IEF increases, presumably due to the sub-auroral polarization stream (SAPS). Another region with enhanced electric fields during large IEF periods is located around 9 MLT at $L>8$, which is possibly related to solar windmagnetosphere coupling. Our potential patterns are consistent with those derived from self-consistent simulations. As the potential patterns can be interpolated/extrapolated to any discrete IEF value within measured ranges, we thus derive an empirical electric potential model. The performance of the model is evaluated by comparing the electric field derived from the model with original one measured by Cluster and mapped to the equator. The model is open to the public through our website.
\end{abstract}

Keywords. Magnetospheric physics (Electric fields; Magnetosphere-ionosphere interactions; Solar windmagnetosphere interactions)

Correspondence to: H. Matsui

(hiroshi.matsui@unh.edu)

\section{Introduction}

The electric field is a key quantity that specifies plasma motion in the inner magnetosphere. The motion of plasmaspheric particles is dominated by the $\boldsymbol{E} \times \boldsymbol{B}$ drift motion (e.g. Carpenter and Seely, 1976; Goldstein et al., 2002; Darrouzet et al., 2006). The motion of ring current particles is dominated by the $\boldsymbol{E} \times \boldsymbol{B}$ drift motion and the gradient $B$ drift motion (e.g. Kistler et al., 1989; Jordanova et al., 2003). The electric field is, in turn, affected by the ring current particles through magnetosphere-ionosphere (M-I) coupling (Vasyliunas, 1970; Jaggi and Wolf, 1973). It is therefore crucial to have accurate information on the electric field in order to understand the dynamics of the inner magnetosphere.

Despite the importance of the electric field to understand the behavior of the plasmasphere and ring current, observations are not so common because measurement of the electric field is not easy. Maynard et al. (1983) reported electric fields inside the plasmasphere from double probe measurements onboard ISEE 1 . There is a strong $K_{p}$ dependence. Electric fields at geosynchronous orbit measured by the electron gun technique were reported by Baumjohann et al. (1985, 1986) and Baumjohann and Haerendel (1985). They showed $K_{p}$, IMF $B_{Z}$, and IMF $B_{Y}$ dependences of the electric fields. They determined the ionospheric shielding effect by analysis of the magnetic local time (MLT) dependence of the electric field. More recently, Rowland and Wygant (1998) reported behavior of the dawn-dusk electric fields from statistical analysis made by CRRES data. The strength of the electric field at large $K_{p}$ index increases as the distance from the Earth decreases so that the radial dependence is opposite to the usual ionospheric shielding of the magnetospheric electric field. Quinn et al. (1999) reported fluctuating electric fields in the inner magnetosphere measured by the Electron Drift Instrument (EDI) on Equator-S. Matsui et al. (2003) analyzed the electric field measured by EDI on Cluster. Various

Published by Copernicus Publications on behalf of the European Geosciences Union. 
types of electric fields exist, such as those originating from solar-wind magnetosphere coupling, subauroral polarization stream (SAPS), and ionospheric dynamo.

Previous electric field models were often developed using plasma data. One of the most widely used models is the Volland-Stern model (Volland, 1973; Stern, 1975). The electric potential is expressed in a simple analytic form: a dawnto-dusk electric field with ionospheric shielding so that the measured shape of the plasmapause can be explained. One parameter found to empirically specify the strength of the dawn-to-dusk electric field is the $K_{p}$ index (Maynard and Chen, 1975). They determined this relation by referring to the location of the plasmapause. Several authors (Jordanova et al., 1999; Ebihara and Ejiri, 2000) introduced direct solar wind control of the Volland-Stern model by using empirical relations between interplanetary parameters and the polar cap (Weimer, 1996; Boyle et al., 1997). McIlwain $(1974,1986)$ derived analytic electric potential models, E3H and E5D models, respectively, from the location of the inner edge of the plasmasheet at geosynchronous orbit. In addition to the above equatorial models, the electric potential models are available at ionospheric height. Heppner and Maynard (1987) derived electric potential patterns by using DE 2 double probe data. Rich and Maynard (1989) later digitized these patterns and fitted them to spherical harmonic functions. The electric potential patterns from DE 2 data were further improved by Weimer $(1995,1996,2001)$. The patterns were expanded by spherical harmonic functions and were organized by interplanetary parameters. The $A L$ index is an optional parametrization, which makes the potential pattern dependent on substorm activity. If we input these controlling parameters to the model, electric field at any ionospheric location at high latitudes can be calculated so that this model is employed in many works (e.g. Elphic et al., 1997; Jordanova et al., 2003).

We have previously investigated statistical electric field patterns in the inner magnetosphere (Matsui et al., 2003, 2004,2005 ) by using electric field data measured by the Electron Drift Instrument (EDI) on Cluster (Paschmann et al., 2001). We inferred that the inner magnetospheric electric field depends on interplanetary parameters. Performing this type of analyses, we noticed that it is worthwhile to develop an inner magnetospheric electric field (UNH-IMEF) model (Puhl-Quinn et al., 2008) because the electric field is a key quantity which specifies particle motion. Simulations in the inner magnetosphere often require electric field as an input parameter. Even if the simulation is performed selfconsistently, it is good to have an empirical model to validate its results. As a first step, Puhl-Quinn et al. (2008) performed merging of the Cluster electric field data from two instruments: EDI and Electric Field and Wave (EFW) instrument (Gustafsson et al., 2001). The availability of the data set is improved by this procedure. In this study, we develop the above-mentioned UNH-IMEF model and examine its performance. The model is used as an input to the ring current-atmosphere interaction model (RAM) to simulate the strength and distribution of the ring current. This part is reported by Jordanova et al. (2008).

This paper is organized as follows. In Sect. 2, our data set is introduced. We describe our analyses and derive average electric field patterns which are sorted by the interplanetary electric field in Sect. 3. An inverse problem is solved to get electric potential patterns from the average electric fields in Sect. 4. We discuss our model in Sect. 5. Our model is compared with previous self-consistent simulation results and with original electric fields measured by Cluster. Finally, conclusions are offered in Sect. 6.

\section{Data}

We use electric field data measured by EDI on Cluster (Paschmann et al., 2001). Two components perpendicular to the ambient magnetic field are available. The instrument measures displacement of electrons due to $\boldsymbol{E} \times \boldsymbol{B}$ drift and slight amount of gradient $B$ drift approximately during one or multiple cycle(s) of cyclotron motion. The test electron beams of energy $1 \mathrm{keV}$, sometimes $500 \mathrm{eV}$, are emitted in the direction perpendicular to the magnetic field. We have occasional data gaps because some particles do not come back to the detectors under conditions with small magnetic field. Some other particles are masked by a large number of ambient electrons such as in the plasmasheet. The inner magnetosphere has a large magnitude of magnetic field so that EDI tends to work well. The maximum time resolution of our data is $1 \mathrm{~s}$ which is set by the ground analysis software. We use data with good quality defined by this software. Data spikes with difference larger than $10 \mathrm{mV} / \mathrm{m}$ from neighboring data are removed. The EFW instrument measures two components of the electric fields in the spacecraft spin plane (Gustafsson et al., 2001). Two pairs of double probe antennas are equipped for each satellite. We obtain this data set from the Cluster Active Archive (CAA) (Lindqvist et al., 2006). The time resolution of our data is $4 \mathrm{~s}$. We use EDI data from SC 1 and SC 3 between February 2001 and October 2006. The data interval for SC 2 is between February 2001 and April 2004 because EDI on SC 2 ceased measurements of electric fields in 2004. EFW data between February 2001 and December 2005 are used, when EDI data are available.

As ring current simulations such as RAM (Jordanova et al., 2003) are often performed in a simulation domain such as $L=2-6.6$, it is necessary to extend our analysis to low $L$ values inside Cluster's perigee, which was $4 R_{E}$ until 2006. We thus introduce electric field data measured by ground incoherent scatter radars and by the DE 2 satellite at the ionospheric height. These are based on previously published statistical results. Ground radar measurements were performed at Millstone Hill with geomagnetic latitude $\lambda=56 \mathrm{de}$ grees (Wand, 1981), Saint-Santin with $\lambda=41$ degrees (Blanc and Amayenc, 1979; Blanc, 1983), and Arecibo with $\lambda=32$ 
degrees (Ganguly et al., 1987). Measurement of azimuthal ion drifts by the DE 2 drift meter was performed at $\lambda=25-65$ degrees (Heelis and Coley, 1992).

The electric field in the inner magnetosphere is sorted by the interplanetary electric field (IEF), which is calculated by using magnetic field and plasma data measured by ACE (Smith et al., 1998; McComas et al., 1998). The IEF is defined as follows:

$\mathrm{IEF}=V \sqrt{B_{Y}^{2}+B_{Z}^{2}} \sin ^{2}(\theta / 2)$,

where $B_{Y}$ and $B_{Z}$ are Y- and Z-components of interplanetary magnetic field (IMF) in the geocentric solar magnetospheric (GSM) coordinates, respectively, and $\theta$ is the IMF clock angle defined $\operatorname{as}^{-1}\left(B_{Y} / B_{Z}\right)$. This definition of IEF is frequently used (e.g. Hairston et al., 2003). The advantage of organizing the inner magnetospheric electric field by an interplanetary parameter lies in its predictive capability because ACE observes interplanetary structures before they arrive at the Earth. Our model could be therefore used to forecast the inner magnetospheric electric field. In our analysis, time lags are taken into account for propagation of structures between the ACE location and the Earth. Averages of the IEF are calculated for $5 \mathrm{~min}, 40 \mathrm{~min}$, and $3 \mathrm{~h}$ to organize the inner magnetospheric electric field, although we mainly use $40 \mathrm{~min}$ averages. The first choice is for instantaneous reaction. The second one is intermediate and also used in our previous studies (Matsui et al., 2003, 2004, 2005). The third one is the same time resolution as the $K_{p}$ index, which is known as a good organizing parameter of the inner magnetospheric electric field (Thomsen, 2004). We will briefly discuss the electric potentials produced using the above three averaging intervals in a later section.

Finally, we introduce the Sym-H index taken from World Data Center at Kyoto University because this is required as an input parameter for the Tsyganenko model (Tsyganenko, 2002), which is used to map electric fields from Cluster locations to the magnetic equator.

\section{Average electric field patterns}

Here we discuss our data analysis and show statistical electric field patterns derived from Cluster data. Other data sets representing the electric field at the ionospheric height are also introduced.

First, it is necessary to merge EDI and EFW data. The details of the process are explained by Puhl-Quinn et al. (2008) so that we describe this procedure briefly. The EDI does not always get return beams as noted, while the EFW has a continuous data coverage. The EFW is sometimes affected by the photoelectron cloud and/or wakes in the vicinity of the spacecraft (Eriksson et al., 2006). The EDI is not much affected by the environment around the spacecraft compared to EFW because the gyroradius of test electron beams is relatively large. For example, the gyroradius of $1 \mathrm{keV}$ electrons is $1.1 \mathrm{~km}$ with an ambient magnetic field of $100 \mathrm{nT}$, while the length of the antennas is $100 \mathrm{~m}$ from tip to tip. The offsets between EDI and EFW are actually measured in the inner magnetosphere (Puhl-Quinn et al., 2008). They also showed that the sampling rate of EDI data decreases as geomagnetic activity increases. Nevertheless, the EDI data tend to be acquired at least once per each $5 \mathrm{~min}$ interval. Thus we have designed the following procedure to create $4 \mathrm{~s}$ resolution merged data. When EDI data are available, we use these data. Otherwise, EFW data are used after correcting for the average offset between EDI and EFW data for each 5 min interval. If there are no EDI data within this interval, EFW data are not included in the merged data. EFW data are also not used when the magnetic field direction is within 15 degrees from the spacecraft spin plane. In this particular case, the spin axis component of the electric field does not have enough accuracy under the assumption of $\boldsymbol{E} \cdot \boldsymbol{B}=0$. As the spin axis is approximately aligned along the $\mathrm{Z}$-axis of the geocentric solar ecliptic (GSE) coordinates, this situation happens at $L \sim 7$ where the geomagnetic field line is approximately in the GSE $X-Y$ plane for Cluster's orbital configuration.

The merged electric field data are further averaged to $5 \mathrm{~min}$ resolution and mapped to the magnetic equatorial plane using the Tsyganenko model (Tsyganenko, 2002). The mapping is performed so that the convective motion of the magnetic field line is consistent between Cluster locations and the magnetic equator. The spatial range we examine for the mapped Cluster data is for $4<L<10$ and for a full range in magnetic local time (MLT). Perigee was located at $L \sim 4$ in our Cluster data set before 2006, and we pick orbits at $L<10$ because we are interested in the inner magnetosphere. Statistics at high latitudes using EDI data were performed by Haaland et al. (2007) and Förster et al. (2007). We also do not want to include data in the magnetosheath. When we check the in situ magnetic field (Balogh et al., 2001) and velocity data, none of the data are obtained with magnetic field strength $B<200 \mathrm{nT}$ and $V_{X}<-30 \mathrm{~km} / \mathrm{s}$. We regard all of the data as measured in the magnetosphere.

Next we divide our database into seven subsets by referring to the IEF values. For each subset, we define a range of IEF values and get one statistical average electric field pattern. Figure 1 shows the relation between accumulated numbers of $5 \mathrm{~min}$ data and IEF values in our database. A large percentage of the data is associated with small IEF values. Although the maximum IEF value is $\sim 16 \mathrm{mV} / \mathrm{m}$, the number of data with IEF $>5 \mathrm{mV} / \mathrm{m}$ is very small so that it is hard to retrieve significant statistical results under these extreme conditions. Nevertheless, we would like to generate our potential patterns at large IEF values. We thus divide our database so that subsets at large (small) IEF values have small (large) number of data. Even if we generate finer subsets at small IEF values, the statistical patterns do not change appreciably. The numbers of $5 \mathrm{~min}$ data points for each subset are noted in Table 1. 
Table 1. Number of $5 \mathrm{~min}$ data points for each subset.

\begin{tabular}{ccccccccc}
\hline IEF & $<0.353$ & $0.353-0.677$ & $0.677-1.024$ & $1.024-1.405$ & $1.405-1.931$ & $1.931-2.690$ & $>2.690$ & Total \\
Number & 9130 & 7987 & 6888 & 5740 & 4624 & 3492 & 2374 & 40235 \\
\hline
\end{tabular}

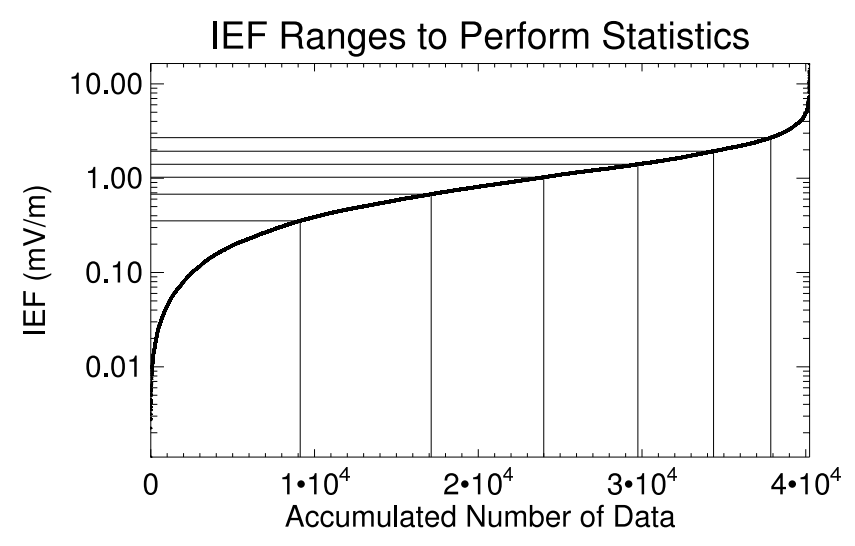

Fig. 1. Accumulated numbers of $5 \mathrm{~min}$ data and IEF are compared in our database. We have divided data into seven categories sorted by IEF.

Figure 2 shows calculated average electric field patterns in the corotating frame for the lowest and highest IEF ranges defined in the previous paragraph. It should be noted that the scale of the electric field vectors is shown in the upper right part of each panel, while the direction of the electric field vectors is rotated 90 degrees clockwise as performed by Matsui et al. (2003) so that the direction of arrows corresponds to that of convection. Each vector is located at $L=4.5-9.5$ with $\Delta L=1$ and 00:30-23:30 MLT with $\Delta M L T=1 \mathrm{~h}$. The electric field during the quiet period is mostly stagnant with the amplitude of at most a few tenths of $1 \mathrm{mV} / \mathrm{m}$. The plasma is corotating at $L \sim 8$ during this quiet condition. The electric field magnitude during the active period is large. The amplitude mostly exceeds $1 \mathrm{mV} / \mathrm{m}$. When we follow the direction of convection from the nightside, this is generally pointing to the dayside corresponding to the solar wind-magnetosphere interaction. These features are consistent with our previous statistics (Matsui et al., 2003).

Finally, we would like to process the electric field data inside the perigee of Cluster. As we noted in the previous section, the published results on ground radar and ionospheric DE 2 measurements are introduced. It should be noted that it is preferable to derive electric fields at $L=1.5-4.5$ with an increment of $\Delta L=1$ from these data in order to keep consistency with the Cluster data analysis. However, the locations of the ground stations are not aligned to these L-values so that we have spatially interpolated or extrapolated the original data. These results are divided into quiet and active conditions by referring to the $K_{p}$ index. Therefore we have to reorganize these electric field data by the IEF. We have investigated the relationship between the IEF and $K_{p}$ index in our database (Fig. 3). Average $K_{p}$ values for each IEF range are calculated and plotted together with standard deviations. Average IEF values and their standard deviations for themselves are also plotted. Although the standard deviations are large, there is a linear relationship between these two parameters. We also calculate average $K_{p}$ values for typical quiet and active conditions, $K_{p} \leq 2$ and $K_{p} \geq 3$, which are indicated by dotted lines. Supplemental electric fields for each IEF range are interpolated or extrapolated from the original electric field values organized by the $K_{p}$ index by referring to the conversion table obtained from this plot. It should be noted that not all of the works specified their quiet and active conditions exactly with the $K_{p}$ ranges noted above. However, we take these $K_{p}$ ranges as representative because the conversion between $K_{p}$ index and IEF is by no means definitive due to large standard deviations in the figure.

Once the above supplemental electric fields are processed, these are merged with the Cluster electric fields. We calculate geometrical means for electric fields at $L=4.5$ from Cluster and these supplemental data, while electric fields at larger and lower L-values are taken from Cluster and these supplemental data, respectively.

\section{Average electric potential patterns}

In this section, we derive electric potential patterns based on the average electric fields explained above. Here we follow procedures noted in Matsui et al. (2004). First we fill data gaps by referring to data at neighboring azimuthal bins. We then use a spatial low-pass filter in which data from original and neighboring bins are averaged. The relation between electric field and potential is given as follows:

$\boldsymbol{E}=\mathbf{A} \phi$,

where $\boldsymbol{E}$ is a vector with two components of the electric fields from all spatial bins, $\mathbf{A}$ is a matrix with spatial gradient operator with a minus sign, and $\boldsymbol{\phi}$ is a vector with electric potentials from all spatial bins. Spatial bins for electric fields are located at $L=1.5-9.5$ and $0.5-23.5$ MLT with $\Delta L=1$ and $\triangle M L T=1 \mathrm{~h}$ with a total number of 216 , so that the dimension of $\boldsymbol{E}$ is twice this, or 432. Spatial bins for the potential are located at $L=1.0-10.0$ and $0.0-23.0$ MLT with a total number of 240 , which is the dimension of $\boldsymbol{\phi}$. We have discarded the potential at $L=1.0$. These bins are shifted by $\Delta L=0.5$ and $\Delta \mathrm{MLT}=0.5 \mathrm{~h}$ from those of the electric field 

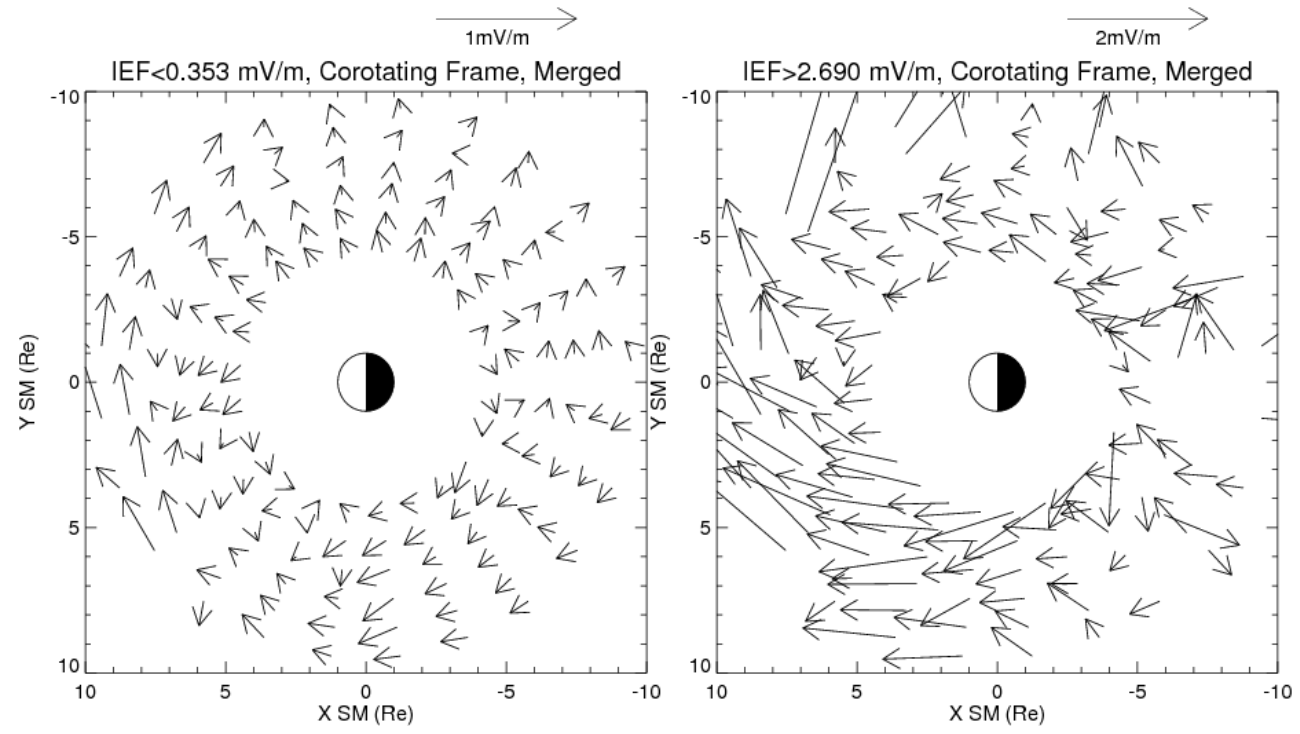

Fig. 2. Average electric field patterns in the corotating frame derived from the Cluster database. The length corresponds to the magnitude of the electric field with a scale shown in the upper right part of each panel. The direction is rotated 90 degrees clockwise, which is the same as the direction of convection. The left and right panels correspond to the patterns for IEF $<0.353 \mathrm{mV} / \mathrm{m}$ and $2.690<\mathrm{IEF} \mathrm{mV} / \mathrm{m}$, respectively.

because of calculation of differentiation. The above equation corresponds to an inverse problem because the calculated quantity $\boldsymbol{\phi}$ is multiplied by a matrix $\mathbf{A}$ to get the original quantity $\boldsymbol{E}$. The estimated potential $\hat{\boldsymbol{\phi}}$ is practically obtained by the following equation:

$\hat{\boldsymbol{\phi}}=\left(\mathbf{A}^{T} \mathbf{A}+\gamma \mathbf{C}^{T} \mathbf{C}\right)^{-1} \mathbf{A}^{T} \boldsymbol{E}$,

where $\gamma$ is a scalar trade-off parameter related to smoothness of the result and $\mathbf{C}$ is a matrix with a Laplacian operator. The choice of $\gamma$ follows a criterion noted in Korth et al. (2002) and Matsui et al. (2004).

Electric potential patterns are calculated for the seven IEF categories as defined above. Four of these potential patterns are shown in Fig. 4. The IEF values increase from Fig. 4a to d. Figure 4a and d corresponds to the patterns at the lowest and highest IEF ranges. In Fig. $4 \mathrm{a}$, the potential difference between maximum and minimum values is $8 \mathrm{kV}$. Equi-potential contours from the nightside deviate around the Earth so that there is shielding of the magnetospheric electric field by the ionosphere. The electric field at low L-values is possibly affected by the ionospheric dynamo because the azimuthal electric field derived from the potential pattern is eastward (westward) in the morning (afternoon) sector, which is a typical feature of this dynamo (e.g. Carpenter and Seely, 1976). The potential contours become denser as the IEF increases (Fig. $4 \mathrm{~b}$ and c). We can see the electric field is getting larger on the duskside than on the dawnside. An example is the electric field at $L \sim 4$ and $\sim 20: 00$ MLT. Some of these large fields are due to subauroral ion drifts (SAID) and/or subauroral polarization stream (SAPS) (Maynard et al., 1980; Rowland and Wygant, 1998;

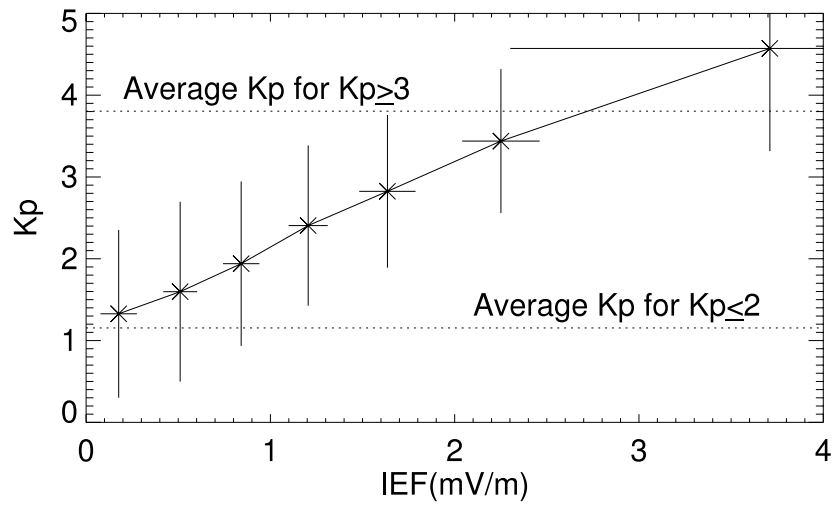

Fig. 3. The relation between IEF and $K_{p}$ index. Average $K_{p}$ values for each IEF range are calculated together with standard deviations shown by error bars. The average $K_{p}$ values for $K_{p} \leq 2$ and $K_{p} \geq 3$ are also indicated by dotted lines.

Anderson et al., 2001; Foster and Vo, 2002). The SAID electric field is actually identified in Cluster data (Puhl-Quinn et al., 2007). The total potential difference is $58 \mathrm{kV}$ in the highest IEF range (Fig. 4d). Strong electric fields are observed at large L-values, and at 09:00 MLT. When we check the original time-series electric field data contributing to this value, we notice that the electric field includes large oscillations. Some of simultaneous particle measurements are examined, typically indicating ion dispersion around the polar cap boundary possibly related to magnetic reconnection. We also find this large and fluctuating electric field on 


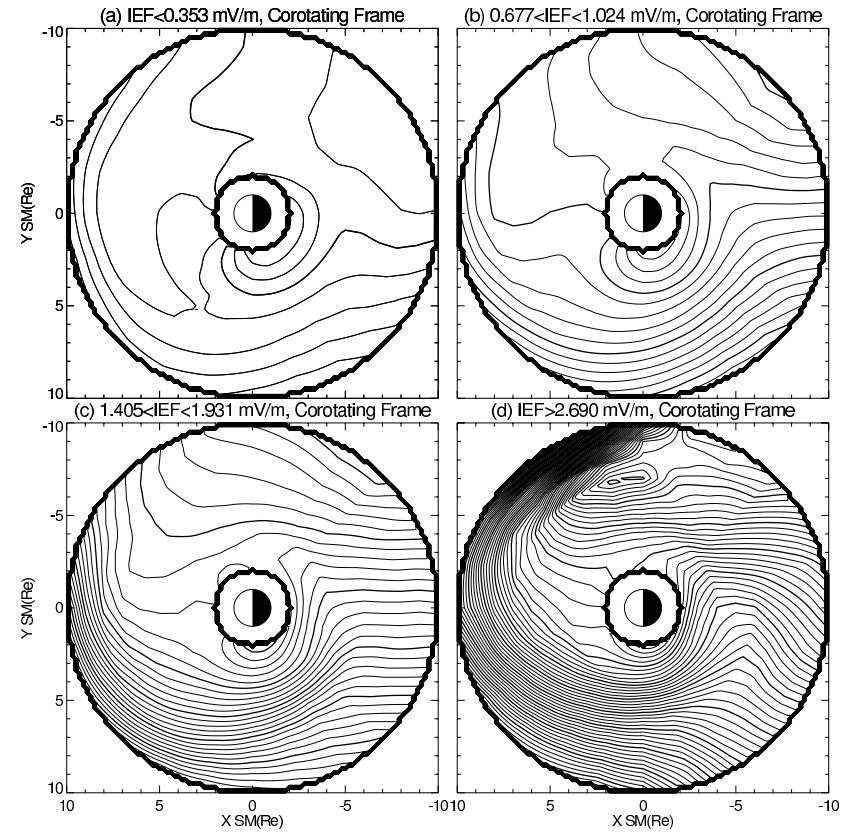

Fig. 4. Calculated electric potential patterns for four IEF ranges: (a) $\mathrm{IEF}<0.353 \mathrm{mV} / \mathrm{m}$, (b) $0.677<\mathrm{IEF}<1.024 \mathrm{mV} / \mathrm{m}$, (c) $1.405<$ IEF $<1.931 \mathrm{mV} / \mathrm{m}$, and (d) $2.690<$ IEF $\mathrm{mV} / \mathrm{m}$. These IEF ranges correspond to first, third, fifth, and seventh ranges in this order from the lowest ranges. The results are shown in corotating frame. Contour intervals are 1 and $5 \mathrm{kV}$ for thin and thick lines, respectively.

the duskside, although such measurement was performed at $L>10$. This observation suggests that direct influence of the solar wind-magnetosphere coupling on the electric field takes place at $L<10$ at $\sim 09: 00 \mathrm{MLT}$, if the mapping procedure is reasonable.

Next we consider interpolation or extrapolation of the seven potential patterns sorted by the IEF. We assume that each potential pattern corresponds to the pattern at an average IEF value within each range. In this case, it is possible to interpolate or extrapolate the potential values at each spatial bin for any selected IEF value. Figure 5 shows such examples of interpolation and extrapolation at two spatial bins. Interpolation is performed between each star, while extrapolation is performed between one star at lowest or highest IEF value and the limit of the IEF value in the figure $(0$ or $5 \mathrm{mV} / \mathrm{m}$ in this example). We apply this procedure to all spatial bins to get electric potential patterns at discrete IEF values we choose. It should be noted that there is a concern regarding this procedure that the offset of the potential value common for all spatial bins is arbitrary for each potential pattern. However, such offset disappears after calculating the spatial gradient to get electric field.

Figure 6 shows examples of interpolated or extrapolated electric potential patterns for $\mathrm{IEF}=0.3,1.5,3$, and $5 \mathrm{mV} / \mathrm{m}$.
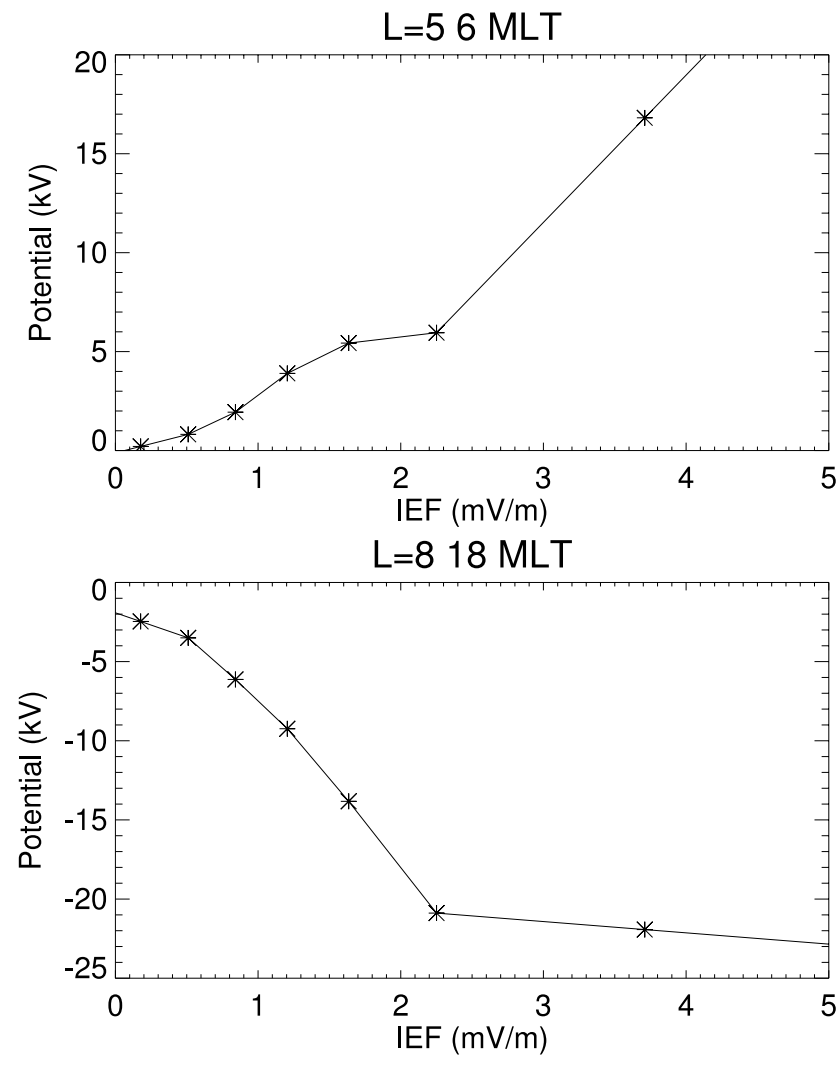

Fig. 5. Interpolation and extrapolation of the electric potentials at two spatial bins. The upper and lower panels show electric potentials at $L=5$ and 06:00 MLT and at $L=8$ and 18:00 MLT, respectively.

The former three patterns are based on interpolation, so that these patterns would give reasonable estimates for the convection patterns. The patterns are more or less similar to the original potential patterns shown in Fig. 4. The last pattern in Fig. 6 is based on extrapolation. This case corresponds to, for example, solar wind velocity of $500 \mathrm{~km} / \mathrm{s}$ and IMF $B_{Z}=-10 \mathrm{nT}$, relatively an active condition. It is necessary to be cautious about interpreting this result. Ring current simulations are often performed during storm periods (e.g. Jordanova et al., 2003). The validity of the extrapolation should be checked by comparing the physical quantities such as the ring current distribution from these simulations and the measurement. Otherwise, we have to consider a way to use sparse data under extreme conditions in which data volume is not large enough to generate potential patterns.

\section{Discussion}

In this section, we would like to show the performance of the model described above. We compare our results with those from self-consistent simulations and with original Cluster 
measurements mapped to the magnetic equator. We also discuss potential difference within the spatial domain of our model derived using three different averaging intervals of IEF: 5, 40, and $180 \mathrm{~min}$.

There are previous works which derived electric potential patterns by self-consistent simulations such as the Rice convection model (RCM) (Garner et al., 2004), combination of RCM and ring current model (Ebihara et al., 2005), and a self-consistent version of ring current-atmosphere interaction model (RAM) (Liemohn et al., 2006). These selfconsistent simulations take into account feedback due to the magnetosphere-ionosphere (M-I) coupling, as proposed by Vasyliunas (1970). It is worthwhile to compare their results with ours. In the simulations, a dominant component of the electric field is the dawn-dusk component caused by the solar wind-magnetosphere coupling. Some of these equipotential contours connecting from the nightside skew toward postmidnight MLT and then come back to the evening side to form the SAID/SAPS structure. In Fig. 4b-d, we also see skewing of the stream lines toward postmidnight MLT, which is a common feature at pre-storm periods and all phases of storms. This indicates qualitative agreement between our model based on measurements, and simulations. It should be noted that the above self-consistent simulations are mostly focused on geomagnetic storm events and that the exact comparison should be made at pre-storm periods. Nevertheless, it is promising to compare more quantitatively the results from experimental and simulation approaches.

Next we evaluate our potential model by comparing with the original electric field which is measured by Cluster (SC 1) and mapped to the equator (Fig. 7). The results shown include data from Cluster's perigee pass at afternoon MLT on 30 July 2001. For original Cluster results, 4-s resolution merged data mapped to the magnetic equator as well as 10 min running averaged data are plotted by small crosses and black solid lines, respectively. We also show electric fields from our model (red lines), Volland-Stern model organized by $K_{p}$ index as Maynard and Chen (1975) specified (green lines), and Weimer model (Weimer, 2001) (blue lines). From this figure, our model and the Cluster observation are actually similar around the perigee between $\sim 15: 10$ 16:30 UT. The interval with good agreement is longer for the radial component than for the azimuthal component. As the spacecraft moves to larger L-value, the discrepancy becomes larger. We also notice that the radial model electric field is generally larger at later time with larger IEF values, which are shown in the top panel. The electric field from the Volland-Stern model is also similar to that from the actual measurement, although the model value tends to be smaller than the measured one. The Weimer model electric field is larger than the observed one, for the radial component.

We also deal with measurements on 13 April 2002 with subauroral structures reported by Matsui et al. (2003) (Fig. 8). The format of this figure is the same as Fig. 7. The spacecraft was at the premidnight sector at $\sim 21: 00$
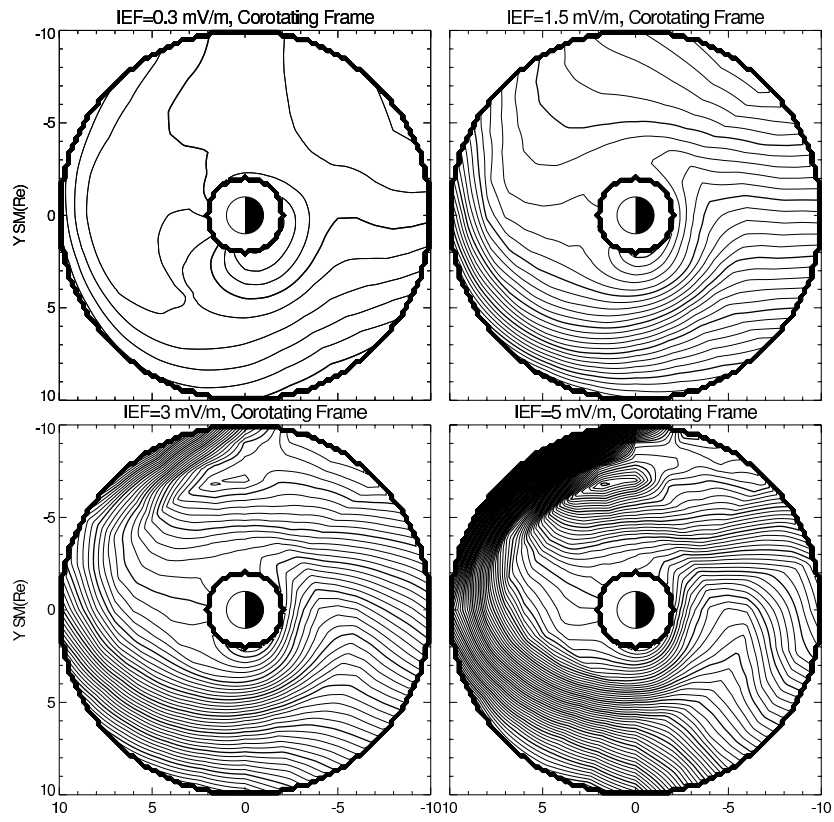

Fig. 6. Interpolated or extrapolated electric potential patterns at $\mathrm{IEF}=0.3,1.5,3$, and $5 \mathrm{mV} / \mathrm{m}$. The format of the figure is the same as Fig. 4.

22:00 MLT. In this case, none of the models reproduce the large electric field in the outward direction as observed. Our model provides closer electric fields to the observation. The original data shown in the figure are apparently included in our statistical database, indicating that the strong subauroral electric field is averaged and smoothed in our model. The improvement of our model perhaps needs accurate information on magnitude and location of the SAID/SAPS structure organized by parameters other than the IEF such as substorm/storm phases. The improvement of the model would be also possible for each event basis by introducing instantaneous measurements. That kind of approach has been adopted by e.g. Richmond (1992) and Ruohoniemi and Baker (1998). Angelopoulos et al. (2002) referred to polar cap potential measured within two hours of interest to improve the electric field estimate by the Weimer (1996) model.

In Fig. 9, we have examined the difference between the model and the original measurement statistically. Standard deviations are calculated by comparing electric fields derived from potential patterns with original ones which are mapped to the equator and averaged for each orbit. Examples of the results at $6<L<7$ and for the whole MLT are chosen for the smallest and largest IEF ranges. The standard deviations are indicated by black lines, while the amplitudes of the electric fields from the model are indicated by red lines. As can be seen, the standard deviation is often larger than the electric fields from the model. This is especially true during the smallest IEF range, although it should 

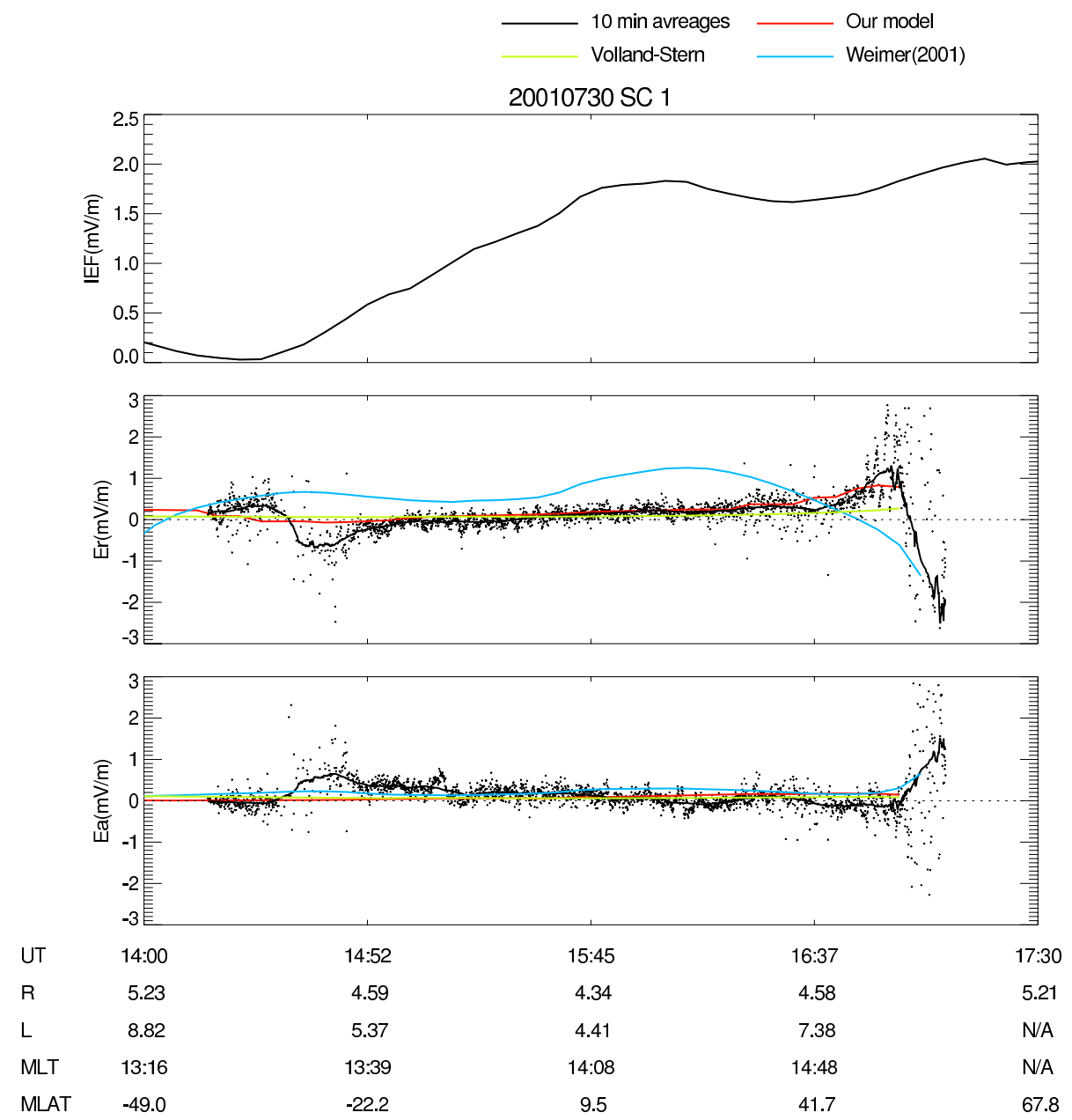

Fig. 7. Comparison between electric fields from original Cluster (SC 1) measurement, its running average, our model, Volland-Stern model, and Weimer (2001) model for 30 July 2001. The panels show the IEF, an input parameter for our model, and radial and azimuthal electric fields. The UT, radial distance $(R)$, L-value, MLT, and magnetic latitude (MLAT) are shown in the bottom. If the L-value and MLT are not available through mapping by the Tsyganenko model, they are indicated as N/A.

be noted that the standard deviation $(\sim 0.2 \mathrm{mV} / \mathrm{m})$ during the smallest IEF is smaller than that $(\sim 0.5-1.5 \mathrm{mV} / \mathrm{m})$ during the largest IEF. If we move from the corotating frame to the inertial frame, the standard deviation during the smallest IEF is getting relatively negligible because the corotation electric field is $\sim 0.7 \mathrm{mV} / \mathrm{m}$ at $6<L<7$. The above result is not much different, even if we choose other L-values. Here it should be noted that we have attempted to remove the contribution of ULF waves on the standard deviation as follows. First we calculate orbital averages of electric fields from 5 min resolution data. Then the standard deviation between the model and the orbital averages are obtained. We get only one average value per one orbit at each L-value and MLT. Thus the large standard deviation would indicate that the electric field is variable between different orbits. Perhaps it is necessary to consider other organizing parameters such as $A L$ index. Further improvement of our model will be left as a future work. Nevertheless, the electric potential patterns show general dependence on the IEF and are qualitatively consistent with self-consistent simulation results.

We compare the electric potential difference in our spatial domain for three averaging intervals for the IEF: 5, 40, and $180 \mathrm{~min}$. The potential differences between maximum and minimum values at $2 \leq L \leq 10$ are calculated in the corotating frame. The results are sorted by the IEF (Fig. 10). The potential differences from three averaging intervals are mostly similar between each other. It is hard to recognize whether there is a saturation effect of the inner magnetospheric electric field during large IEF periods in Fig. 10. One possible reason is that we rarely measure extreme geomagnetic conditions. Hairston et al. (2003) discussed saturation events with IEF $>10 \mathrm{mV} / \mathrm{m}$, while the IEF range in this figure is $<5 \mathrm{mV} / \mathrm{m}$. 

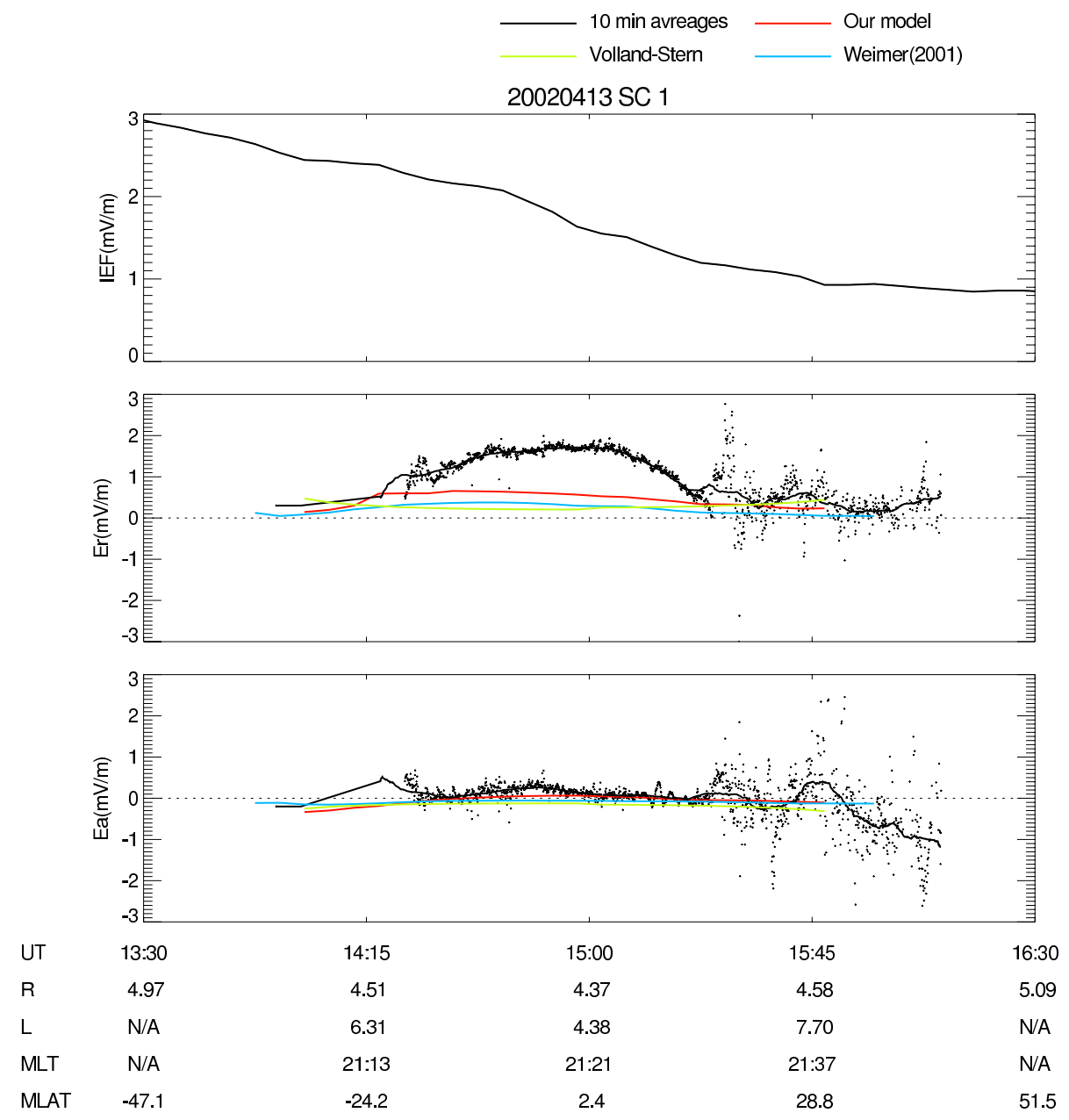

Fig. 8. Comparison between electric fields from original Cluster (SC 1) measurement, its running average, our model, Volland-Stern model, and Weimer (2001) model for 13 April 2002. The format of this figure is the same as Fig. 7.

In our analysis, we organize electric potential patterns by IEF with fixed time averages. However, there are various time scales for the electric field at low L-values, which are partially averaged out in our statistics. The electric field with short time scales result from prompt penetration of magnetospheric electric fields (Senior and Blanc, 1984). The strength of the penetration electric field depends on the time rate of change of the IEF (Senior and Blanc, 1984; Peymirat et al., 2000). The electric field with longer time scales result from disturbance winds driven by high-latitude activity (Blanc and Richmond, 1980). These effects should be carefully taken into account in future update of the model.

Finally, we have evaluated the induction electric fields in our database. We have calculated eastward electric fields averaged for whole MLT for each L-value $(L=4.5-9.5)$. The statistical data at the largest IEF range (IEF $>2.690 \mathrm{mV} / \mathrm{m}$ ) are chosen because these data are expected to include data from storm main phase. The ring current is strengthening and the field is inflating during this storm phase so that an eastward induction electric field is expected at all MLT. The average eastward electric fields for whole MLT and for each L-value are $0.06-0.51 \mathrm{mV} / \mathrm{m}$, in which the average values tend to increase as the L-value increases. The results at the largest IEF range is significant compared to those from whole of our database $(0.03-0.08 \mathrm{mV} / \mathrm{m}$ depending on L-value).

\section{Conclusions}

In this study, we have developed an inner magnetospheric electric field (UNH-IMEF) model at $2 \leq L \leq 10$. We introduce Cluster data for more than five years. Merged data are generated from complementary EDI and EFW instruments. Our database is further supplemented by the ground radar and DE 2 satellite data. The inner magnetospheric electric field is sorted by the interplanetary electric field (IEF). Electric potential patterns for seven IEF categories are calculated from the average electric field patterns by solving an inverse 
Standard Deviation

E calcuated from Phi
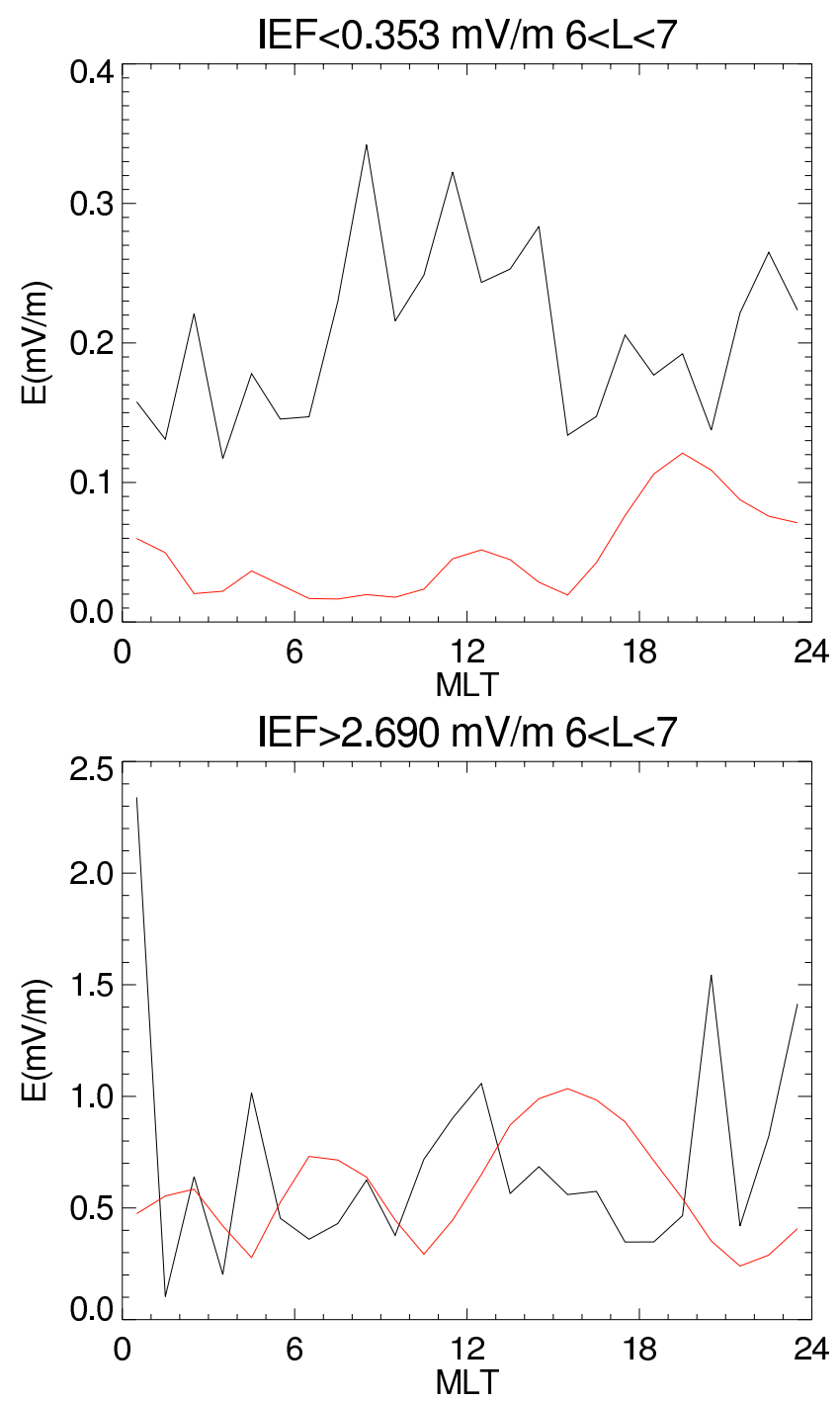

Fig. 9. Standard deviation between electric fields from our model and those from the original measurement mapped to the equator is shown by black lines. The magnitude of electric field from our model is shown by red lines. Top and bottom panels show the results at the smallest and largest IEF ranges, respectively. The results at $6<L<7$ are organized by MLT which is indicated in the horizontal axis.

problem. Then electric potential patterns for any IEF values can be interpolated or extrapolated from the above seven potential patterns. The developed model is qualitatively consistent with self-consistent simulation results. The model is further evaluated by comparing with the original electric fields measured by Cluster as well as a few other models. The standard deviation between the electric field from the model and that from the observation is comparable to or larger than

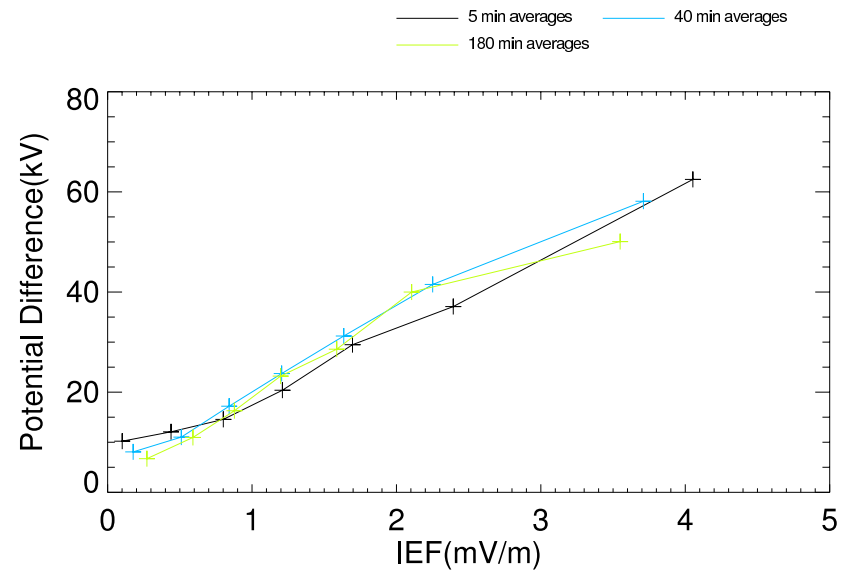

Fig. 10. The relation between IEF and potential difference at $2 \leq L \leq 10$ in our statistics. Potential differences are calculated for three averaging intervals of the IEF: 5, 40, and $180 \mathrm{~min}$.

the amplitude of the electric field from the model so that the model still requires further improvement. Nevertheless, this model would be useful for simulations of plasmasphere and/or ring current. Our model is available at the following website (http://edi.sr.unh.edu/unh-imef/).

We can consider the following future works to validate and improve our model. As for one validation, Jordanova et al. (2008) drive their RAM simulation model by using our model and compare their outputs with measurements. Extension of such study is necessary to get feedbacks to improve the model. As for the improvement, Cluster decrease their perigee from $4 R_{E}$ continuously since 2006 until the spacecraft finally clashes into the atmosphere in 2009. These low- $L$ data will be useful to improve our model at $2<L<4$, where we now use data from ionospheric measurements. We are also examining data from the Cluster Ion Spectrometry (CIS) instrument. This would improve our data coverage especially during substorm injections. In addition, it is necessary to think about the procedure to improve the estimation of the electric field by combining this model with the simultaneous measurements, which would reduce the difference between measurements and the model for each event basis.

Acknowledgements. We thank Cluster Active Archive (CAA) for double probe data. We are grateful to E. A. Lucek and H. Rème for magnetic field data and energy-time spectra from Cluster, respectively. N. F. Ness and D. J. McComas provided ACE MAG and SWEPAM data through CDA Website, respectively. Sym-H index is taken from World Data Center at Kyoto University. This work was supported by NASA through grant NNG05GG50G.

Topical Editor R. Nakamura thanks two anonymous referees for their help in evaluating this paper. 


\section{References}

Anderson, P. C., Carpenter, D. L., Tsuruda, K., Mukai, T., and Rich, F. J.: Multisatellite observations of rapid subauroral ion drifts (SAID), J. Geophys. Res., 106, 29 585-29 599, 2001.

Angelopoulos, V., Temerin, M., Roth, I., Mozer, F. S., Weimer, D., and Hairston, M. R.: Testing global storm-time electric field models using particle spectra on multiple spacecraft, J. Geophys. Res., 107(A8), 1194, doi:10.1029/2001JA900174, 2002.

Balogh, A., Carr, C. M., Acuña, M. H., et al.: The Cluster Magnetic Field Investigation: overview of in-flight performance and initial results, Ann. Geophys., 19, 1207-1217, 2001, http://www.ann-geophys.net/19/1207/2001/.

Baumjohann, W. and Haerendel, G.: Magnetospheric convection observed between 0600 and 2100 LT: Solar wind and IMF dependence, J. Geophys. Res., 90, 6370-6378, 1985.

Baumjohann, W., Haerendel, G., and Melzner, F.: Magnetospheric convection observed between 0600 and 2100 LT: Variations with $K_{p}$, J. Geophys. Res., 90, 393-398, 1985.

Baumjohann, W., Nakamura, R., and Haerendel, G.: Dayside equatorial-plane convection and IMF sector structure, J. Geophys. Res., 91, 4557-4560, 1986.

Blanc, M.: Magnetospheric convection effects at mid-latitude 1. Saint-Santin observations, J. Geophys. Res., 88, 211-223, 1983.

Blanc, M. and Amayenc, P.: Seasonal variations of the ionospheric $E \times B$ drifts above Saint-Santin on quiet days, J. Geophys. Res., 84, 2691-2704, 1979.

Blanc, M. and Richmond, A. D.: The ionospheric disturbance dynamo, J. Geophys. Res., 85, 1669-1686, 1980.

Boyle, C. B., Reiff, P. H., and Hairston, M. R.: Empirical polar cap potentials, J. Geophys. Res., 102, 111-125, 1997.

Carpenter, D. L. and Seely, N. T.: Cross- $L$ plasma drifts in the outer plasmasphere: Quiet time patterns and some substorm effects, J. Geophys. Res., 81, 2728-2736, 1976.

Darrouzet, F., De Keyser, J., Décréau, P, M. E., et al.: Analysis of plasmaspheric plumes: CLUSTER and IMAGE observations, Ann. Geophys., 24, 1737-1758, 2006, http://www.ann-geophys.net/24/1737/2006/.

Ebihara, Y. and Ejiri, M.: Simulation study on fundamental properties of the storm-time ring current, J. Geophys. Res., 105, 15 843-15 859, 2000.

Ebihara, Y., Fok, M.-C., Sazykin, S., Thomsen, M. F., Hairston, M. R., Evans, D. S., Rich, F. J., and Ejiri, M.: Ring current and the magnetosphere-ionosphere coupling during the superstorm of 20 November 2003, J. Geophys. Res., 110, A09S22, doi:10.1029/2004JA010924, 2005.

Elphic, R. C., Thomsen, M. F., and Borovsky, J. E.: The fate of the outer plasmasphere, Geophys. Res. Lett., 24, 365-368, 1997.

Eriksson, A. I., André, M., Klecker, B., et al.: Electric field measurements on Cluster: comparing the double-probe and electron drift techniques, Ann. Geophys., 275-289, 2006.

Förster, M., Paschmann, G., Haaland, S. E., Quinn, J. M., Torbert, R. B., Vaith, H., and Kletzing, C. A.: High-latitude plasma convection from Cluster EDI: variances and solar wind correlations, Ann. Geophys., 25, 1691-1707, 2007, http://www.ann-geophys.net/25/1691/2007/.

Foster, J. C. and Vo, H. B.: Average characteristics and activity dependence of the subauroral polarization stream, J. Geophys. Res., 107(A12), 1475, doi:10.1029/2002JA009409, 2002.

Ganguly, S., Behnke, R. A., and Emery, B. A.: Average electric field behavior in the ionosphere above Arecibo, J. Geophys. Res., 92, 1199-1210, 1987.

Garner, T. W., Wolf, R. A., Spiro, R. W., Burke, W. J., Fejer, B. G., Sazykin, S., Roeder, J. L., and Hairston, M. R.: Magnetospheric electric fields and plasma sheet injection to low L-shells during the 4-5 June 1991 magnetic storm: Comparison between the Rice Convection Model and observations, J. Geophys. Res., 109, A02214, doi:10.1029/2003JA010208, 2004.

Goldstein, J., Spiro, R. W., Reiff, P. H., Wolf, R. A., Sandel, B. R., Freeman, J. W., and Lambour, R. L.: IMF-driven overshielding electric field and the origin of the plasmaspheric shoulder of May 24, 2000, Geophys. Res. Lett., 29(16), 1819, doi:10.1029/2001GL014534, 2002.

Gustafsson, G., André, M., Carozzi, T., et al.: First results of electric field and density observations by Cluster EFW based on initial months of operation, Ann. Geophys., 19, 1219-1240, 2001, http://www.ann-geophys.net/19/1219/2001/.

Haaland, S. E., Paschmann, G., Förster, M., Quinn, J. M., Torbert, R. B., McIlwain, C. E., Vaith, H., Puhl-Quinn, P. A., and Kletzing, C. A.: High-latitude plasma convection from Cluster EDI measurements: method and IMF-dependence, Ann. Geophys., 25, 239-253, 2007,

http://www.ann-geophys.net/25/239/2007/.

Hairston, M. R., Hill, T. W., and Heelis, R. A.: Observed saturation of the ionospheric polar cap potential during the 31 March 2001 storm, Geophys. Res. Lett., 30(6), 1325, doi:10.1029/2002GL015894, 2003.

Heelis, R. A. and Coley, W. R.: East-west ion drifts at mid-latitudes observed by Dynamics Explorer 2, J. Geophys. Res., 97, 1946119469, 1992.

Heppner, J. P. and Maynard, N. C.: Empirical high-latitude electric field models, J. Geophys. Res., 92, 4467-4489, 1987.

Jaggi, R. K. and Wolf, R. A.: Self-consistent calculation of the motion of a sheet of ions in the magnetosphere, J. Geophys. Res., 78, 2852-2866, 1973.

Jordanova, V. K., Torbert, R. B., Thorne, R. M., Collin, H. L., Roeder, J. L., and Foster, J. C.: Ring current activity during the early $B_{z}<0$ phase of the January 1997 magnetic cloud, J. Geophys. Res., 104, 24 895-24 914, 1999.

Jordanova, V. K., Boonsiriseth, A., Thorne, R. M., and Dotan, Y.: Ring current asymmetry from global simulations using a high resolution electric field model, J. Geophys. Res., 108(A12), 1443, doi:10.1029/2003JA009993, 2003.

Jordanova, V. K., Matsui, H., Puhl-Quinn, P. A., Thomsen, M. F., Mursula, K., and Holappa, L.: Ring current development during high speed streams, J. Atmos. Solar-Terr. Phys., submitted, 2008.

Kistler, L. M., Ipavich, F. M., Hamilton, D. C., Gloeckler, G., Wilken, B., Kremser, G., and Stüdemann, W.: Energy spectra of the major ion species in the ring current during geomagnetic storms, J. Geophys. Res., 94, 3579-3599, 1989.

Korth, H., Thomsen, M. F., Glassmeier, K.-H., and Phillips, W. S.: Particle tomography of the inner magnetosphere, J. Geophys. Res., 107(A9), 1229, doi:10.1029/2001JA000147, 2002.

Liemohn, M. W., Ridley, A. J., Kozyra, J. U., Gallagher, D. L., Thomsen, M. F., Henderson, M. G., Denton, M. H., Brandt, P. C., and Goldstein, J.: Analyzing electric field morphology through data-model comparisons of the Geospace Environment Modeling Inner Magnetosphere/Storm Assessment Challenge events, J. Geophys. Res., 111, A11S11, doi:10.1029/2006JA011700, 2006. 
Lindqvist, P.-A., Khotyaintsev, Y., André, M., and Eriksson, A. I.: EFW data in the Cluster Active Archive, Proceedings Cluster and Double Star Symposium, ESA SP-598, 2006.

Matsui, H., Quinn, J. M., Torbert, R. B., Jordanova, V. K., Baumjohann, W., Puhl-Quinn, P. A., and Paschmann, G.: Electric field measurements in the inner magnetosphere by Cluster EDI, J. Geophys. Res., 108(A9), 1352, doi:10.1029/2003JA009913, 2003

Matsui, H., Jordanova, V. K., Quinn, J. M., Torbert, R. B., and Paschmann, G.: Derivation of electric potential patterns in the inner magnetosphere from Cluster EDI data: Initial results, J. Geophys. Res., 109, A10202, doi:10.1029/2003JA010319, 2004.

Matsui, H., Quinn, J. M., Torbert, R. B., Jordanova, V. K., PuhlQuinn, P. A., and Paschmann, G.: IMF $\boldsymbol{B}_{\boldsymbol{Y}}$ and seasonal dependences of the electric field in the inner magnetosphere, Ann. Geophys., 23, 2671-2678, 2005, http://www.ann-geophys.net/23/2671/2005/.

Maynard, N. C. and Chen, A. J.: Isolated cold plasma regions: Observations and their relation to possible production mechanisms, J. Geophys. Res., 80, 1009-1013, 1975.

Maynard, N. C., Aggson, T. L., and Heppner, J. P.: Magnetospheric observation of large sub-auroral electric fields, Geophys Res. Lett., 7, 881-884, 1980.

Maynard, N. C., Aggson, T. L., and Heppner, J. P.: The plasmaspheric electric field as measured by ISEE 1, J. Geophys. Res., 88, 3991-4003, 1983.

McComas, D. J., Bame, S. J., Baker, P., Feldman, W. C., Phillips, J. L., Riley, P., and Griffee, J. W.: Solar wind electron proton alpha monitor (SWEPAM) for the advanced composition explorer, Space Sci. Rev., 86, 563-612, 1998.

McIlwain, C. E.: Substorm injection boundaries, in Magnetospheric Physics, edited by: McCormac, B. M., pp. 143-154, D. Reidel, Dordrecht, Netherlands, 1974.

McIlwain, C. E.: A $K_{p}$ dependent equatorial electric field model, Adv. Space Res., 6(3), 187-197, 1986.

Paschmann, G., Quinn, J. M., Torbert, R. B., et al.: The Electron Drift Instrument on Cluster: overview of first results, Ann Geophys., 19, 1273-1288, 2001.

Peymirat, C., Richmond, A. D., and Kobea, A. T.: Electrodynamic coupling of high and low latitudes: Simulations of shielding/overshielding effects, J. Geophys. Res., 105, 22 991-23 003, 2000.

Puhl-Quinn, P. A., Matsui, H., Mishin, E., Mouikis, C., Kistler, L, Khotyaintsev, Y., Décréau, P. M. E., and Lucek, E.: Cluster and DMSP observations of SAID electric fields, J. Geophys. Res., 112, A05219, doi:10.1029/2006JA012065, 2007.

Puhl-Quinn, P. A., Matsui, H., Jordanova, V. K., Khotyaintsev, Y., and Lindqvist, P.-A.: An effort to derive an empirically based, inner-magnetospheric electric field model: Merging Cluster EDI and EFW data, J. Atmos. Solar-Terr. Phys., 70, 564-573, 2008.
Quinn, J. M., Paschmann, G., Sckopke, N., et al.: EDI convection measurements at $5-6 R_{E}$ in the post-midnight region, Ann. Geophys., 17, 1503-1512, 1999, http://www.ann-geophys.net/17/1503/1999/.

Rich, F. J. and Maynard, N. C.: Consequences of using simple analytical functions for the high-latitude convection electric field, J. Geophys Res., 94, 3687-3701, 1989.

Richmond, A. D.: Assimilative mapping of ionospheric electrodynamics, Adv. Space Res., 12(6), 59-68, 1992.

Rowland, D. E. and Wygant, J. R.: Dependence of the large-scale, inner magnetospheric electric field on geomagnetic activity, J. Geophys. Res., 103, 14 959-14 964, 1998.

Ruohoniemi, J. M. and Baker, K. B.: Large-scale imaging of highlatitude convection with Super Dual Auroral Radar Network HF radar observations, J. Geophys. Res., 103, 20 797-20 811, 1998.

Senior, C. and Blanc, M.: On the control of magnetospheric convection by the spatial distribution of ionospheric conductivities, J. Geophys. Res., 89, 261-284, 1984.

Smith, C. W., L'Heureux, J., Ness, N. F., Acuña, M. H., Burlaga, L. F., and Scheifele, J.: The ACE magnetic fields experiment, Space Sci. Rev., 86, 613-632, 1998.

Stern, D. P.: The motion of a proton in the equatorial magnetosphere, J. Geophys. Res. 80, 595-599, 1975.

Thomsen, M. F.: Why $K_{p}$ is such a good measure of magnetospheric convection, Space Weather, 2, S11004, doi:10.1029/2004SW000089, 2004.

Tsyganenko, N. A.: A model of the near magnetosphere with a dawn-dusk asymmetry 1. Mathematical structure, J. Geophys. Res., 107(A8), 1179, doi:10.1029/2001JA000219, 2002.

Vasyliunas, V. M.: Mathematical models of magnetospheric convection and its coupling to the ionosphere, in: Particles and Fields in the Magnetosphere, edited by: McCormac, B. M., D Reidel, Dordrecht, Netherlands, 60-71, 1970.

Volland, H.: A semiempirical model of large-scale magnetospheric electric fields, J. Geophys. Res., 78, 171-180, 1973.

Wand, R. H.: A model representation of the ionospheric electric field over Millstone Hill $\left(\lambda=56^{\circ}\right)$, J. Geophys. Res., 86, 58015808, 1981.

Weimer, D. R.: Models of high-latitude electric potentials derived with a least error fit of spherical harmonic coefficients, J. Geophys. Res., 100, 19595-19607, 1995.

Weimer, D. R.: A flexible, IMF dependent model of high-latitude electric potentials having "space weather" applications, Geophys. Res. Lett., 23, 2549-2552, 1996.

Weimer, D. R.: An improved model of ionospheric electric potentials including substorm perturbations and applications to the Geospace Environment Modeling November 24, 1996, event, J. Geophys. Res., 106, 407-416, 2001. 